2016-07-01

\title{
Disrupting Hypoxia-Induced Bicarbonate Transport Acidifies Tumor Cells and Suppresses Tumor Growth
}

\author{
Mclntyre, A
}

http://hdl.handle.net/10026.1/5238

10.1158/0008-5472.CAN-15-1862

Cancer Research

All content in PEARL is protected by copyright law. Author manuscripts are made available in accordance with publisher policies. Please cite only the published version using the details provided on the item record or document. In the absence of an open licence (e.g. Creative Commons), permissions for further reuse of content should be sought from the publisher or author. 


\title{
Disrupting Hypoxia-Induced Bicarbonate Transport Acidifies Tumor Cells and Suppresses Tumor Growth
}

\author{
Alan McIntyre ${ }^{1,2}$, Alzbeta Hulikova ${ }^{3}$, Ioanna Ledaki ${ }^{1}$, Cameron Snell ${ }^{4}$, \\ Dean Singleton', Graham Steers', Peter Seden ${ }^{5,6}$, Dylan Jones ${ }^{1}$, \\ Esther Bridges', Simon Wigfield', Ji-Liang Li', Angela Russell ${ }^{5,6}$ \\ Pawel Swietach ${ }^{3}$, and Adrian L. Harris ${ }^{1}$
}

\section{Abstract}

Tumor hypoxia is associated clinically with therapeutic resistance and poor patient outcomes. One feature of tumor hypoxia is activated expression of carbonic anhydrase IX (CA9), a regulator of $\mathrm{pH}$ and tumor growth. In this study, we investigated the hypothesis that impeding the reuptake of bicarbonate produced extracellularly by CA9 could exacerbate the intracellular acidity produced by hypoxic conditions, perhaps compromising cell growth and viability as a result. In 8 of 10 cancer cell lines, we found that hypoxia induced the expression of at least one bicarbonate transporter. The most robust and frequent inductions were of the sodium-driven bicarbonate transporters SLC4A4 and SLC4A9, which rely upon both HIF1 $\alpha$ and HIF2 $\alpha$ activity for their expres- sion. In cancer cell spheroids, SLC4A4 or SLC4A9 disruption by either genetic or pharmaceutical approaches acidified intracellular $\mathrm{pH}$ and reduced cell growth. Furthermore, treatment of spheroids with S0859, a small-molecule inhibitor of sodium-driven bicarbonate transporters, increased apoptosis in the cell lines tested. Finally, RNAi-mediated attenuation of SLC4A9 increased apoptosis in MDA-MB-231 breast cancer spheroids and dramatically reduced growth of MDA-MB-231 breast tumors or U87 gliomas in murine xenografts. Our findings suggest that disrupting $\mathrm{pH}$ homeostasis by blocking bicarbonate import might broadly relieve the common resistance of hypoxic tumors to anticancer therapy. Cancer Res; 76(13); 3744-55. @2016 AACR.

\section{Introduction}

Hypoxia (low oxygen) and acidosis are key physiologica features commonly associated with solid tumors. Hypoxia results from high metabolic demand and proliferative rates, combined with poor tumor perfusion. These factors increase oxygen requirements while reducing oxygen availability (1). Clinically, hypoxia is associated with poor patient prognosis and resistance to chemotherapy and radiotherapy (2). Developing new strategies to target the hypoxic microenvironment is critical for improving

${ }^{1}$ Molecular Oncology Laboratories, Department of Oncology, University of Oxford, Weatherall Institute of Molecular Medicine, Oxford, United Kingdom. ${ }^{2}$ Cancer Biology, Division of Cancer and Stem Cells, University of Nottingham, Nottingham, United Kingdom. ${ }^{3}$ Department of Physiology, Anatomy and Genetics, University of Oxford Oxford, United Kingdom. ${ }^{4}$ Nuffield Department of Clinical Laboratory Sciences, Radcliffe Department of Medicine, University of Oxford, Oxford, United Kingdom. ${ }^{5}$ Chemistry Research Laboratory, Department of Chemistry, University of Oxford, Oxford, United Kingdom. ${ }^{6}$ Department of Pharmacology, University of Oxford, Oxford, United Kingdom.

Note: Supplementary data for this article are available at Cancer Research Online (http://cancerres.aacrjournals.org/).

Corresponding Author: Adrian L. Harris, University of Oxford, Molecular Oncology Laboratories, Department of Oncology, Weatherall Institute of Molecular Medicine, John Radcliffe Hospital, Oxford OX3 9DS, United Kingdom. Phone: 018-6522-2457; Fax: 018-6522-2431; E-mail: aharris.lab@imm.ox.ac.uk

doi: 10.1158/0008-5472.CAN-15-1862

(C)2016 American Association for Cancer Research. patient outcome. Furthermore, antiangiogenic therapy induces tumor hypoxia in approximately half of cases (where the remainder of the tumors exhibit no vascular response or vascular normalization for a period at the start of therapy), and combination strategies that induce apoptosis in the hypoxic microenvironment would be effective in this context (3). Hypoxia results in stabilization of the transcription factors hypoxia inducible factors (HIF) $1 \alpha$ and $2 \alpha$, via reduced hydroxylation of HIF proteins, preventing ubiquitination pVHL (protein Von Hippel-Lindau) which precedes HIF proteasomal degradation (4). Many HIFregulated genes trigger more aggressive tumor growth, invasion, metabolic adaptation, and survival (3).

The extracellular microenvironment of hypoxic tumors is acidic (5) because of increased production of metabolic acids $\left(\mathrm{CO}_{2}\right.$ and lactic acid) and longer diffusion distances to the nearest functional blood capillary for acid venting. A mismatch between acid production and venting can profoundly change steady-state intra- and extracellular $\mathrm{pH}$, which could have major effects on cell survival because $\mathrm{H}^{+}$ions are highly reactive and target essentially all protein-dependent processes (6). This is exacerbated by the fact that the optimal range of intracellular $\mathrm{pH}\left(\mathrm{pH}_{\mathrm{i}}\right)$ is very narrow. In most cells, a mildly alkaline $\mathrm{pH}_{\mathrm{i}}$ supports growth and function, and this condition is normally maintained by transporter proteins at the cell membrane that handle $\mathrm{H}^{+}$ions or their chemical equivalents (e.g., $\mathrm{HCO}_{3}{ }^{-}$or $\mathrm{OH}^{-}$). This homeostatic mechanism is challenged by the low extracellular $\mathrm{pH}\left(\mathrm{pH}_{\mathrm{e}}\right)$ of tumors because extracellular acidity can reduce acid-extrusion flux allosterically (7) and thermodynamically (i.e., pumping against a steeper $\left[\mathrm{H}^{+}\right]$gradient). The 
$\mathrm{pH}_{\mathrm{i}}$-regulatory apparatus in cancer cells must therefore maintain a transmembrane $\mathrm{pH}$ gradient through active transport of adequate flux magnitude (6). The characteristic distribution of $\mathrm{H}^{+}$ions across tumor aqueous compartments is dually favorable for cancer progression. While the mildly alkaline $\mathrm{pH}_{\mathrm{i}}$ supports proliferation and reduces sensitivity to apoptosis, the more acidic $\mathrm{pH}_{\mathrm{e}}$ favors increased extracellular matrix degradation, facilitating invasion and metastasis $(8,9)$.

HIF1 $\alpha$-regulated CA9 (10) facilitates $\mathrm{CO}_{2}$ venting from diffusion-limited tissue by augmenting its extracellular diffusion as its hydration products $\left(\mathrm{H}^{+}\right.$and $\mathrm{HCO}_{3}{ }^{-}$; ref. 11). In spheroid models, we have demonstrated that this CA9-driven mechanism regulates the intracellular and extracellular $\mathrm{pH}$ (12). Clinically, CA9 is a marker for poor clinical outcome in most cancer types (13-16) and recent work has identified the importance of CA9 in tumor growth, metastasis, and response to antiangiogenic therapy (17-19). However, in order for CA9 to operate in the direction of net hydration, cells must release $\mathrm{CO}_{2}(20)$. Under hypoxic conditions, this requires cellular uptake of bicarbonate, which can be sourced from the extracellular CA9 reaction. The bicarbonate uptake process represents the active transport mechanism that drives uphill acid efflux, and is therefore essential for $\mathrm{pH}$ homeostasis, an inherently energy-demanding process.

Bicarbonate transporters are grouped into the SLC4 and SLC26 protein families (21-23). These can be subdivided into two classes. The $\mathrm{Na}^{+}$-independent anion exchangers include SLC4A 1-3 and SLC26A 2-4, 7, 9, and 11, and many of these proteins are nominally acid loaders. The second major class is the $\mathrm{Na}^{+}$-driven bicarbonate transporters (NDBT), including $\mathrm{Na}^{+}-\mathrm{HCO}_{3}{ }^{-}$cotransporters (NBC) SLC4A4 (electrogenic NBCe1), SLC4A5 (electrogenic NBCe2), SLC4A7 (electroneutral NBCn1), and SLC4A10 (NBCn2), and the $\mathrm{Na}^{+}$-driven anion exchangers SLC4A8 $\left(\mathrm{Na}^{+}-\right.$ driven $\mathrm{Cl}^{-} / \mathrm{HCO}_{3}{ }^{-}$exchange) and, putatively, SLC4A9 (AE4; refs. 21, 24). SLC4A4 and 7 are well-characterized acid extruders. SLC4A9 has been controversial, with differing reports of its functional role, possibly because of the use of the SLC4A9 gene from different species in heterologous expression systems (21). Amino acid sequence analysis showed that SLC4A9 has substantial similarity with the NBCs (21). Functional studies of human SLC4A9 showed it produced a $\mathrm{Na}^{+}$-driven $\mathrm{pH}_{\mathrm{i}}$ recovery from a $\mathrm{CO}_{2}$-induced acidosis (24).

In several cancer cell lines, we showed a shift in pH regulation toward bicarbonate-dependent transport occurs in hypoxia (25). The importance of $\mathrm{pH}$ regulation for survival in the hypoxic tumor microenvironment presents an opportunity to induce synthetic lethality by disrupting acid/base balance. Hypoxia has been shown to increase SLC4A4 expression in the Ls174T colon cancer cell line (26). That study also demonstrated that knockdown of SLC4A4 slowed the recovery of intracellular $\mathrm{pH}$ from an intracellular acid load, reduced twodimensional (2D) growth, increased cell mortality in acidic conditions, and reduced spheroid growth (26). Intriguingly, increased levels of bicarbonate transporters have been reported in several types of tumors $(27,28)$.

Here, we investigated the expression of all major bicarbonate transporters of the SLC4 and SLC26 families in response to hypoxia. We examined the role of HIF in hypoxic induction of bicarbonate transporters. We analyzed the specific role of hypoxia-induced SLC4A9. The physiologic role of this protein has not been recognized in cancer, and we present data for its critical role in $\mathrm{pH}$ regulation and growth. We show that SLC4A9 is important for spheroid growth, $\mathrm{pH}$ regulation and survival, and growth of xenografts in vivo.

Furthermore, to inhibit bicarbonate transport pharmacologically, we used S0859, a drug developed over a decade ago as an inhibitor of sodium dependent bicarbonate co-transporters. This small molecule inhibitor blocks the bulk of acid extrusion carried by sodium-driven bicarbonate transport $(7,29,30)$, but other proteins have been shown to be sensitive to S0859 including lactate transporters ectopically expressed in oocytes (31). We used S0859 to explore the role of sodium driven bicarbonate transporters on 3D spheroid growth and intracellular $\mathrm{pH}$ regulation. This study demonstrates the importance of bicarbonate transport in $\mathrm{pH}$ homeostasis and tumor growth, and identifies new molecular targets that disrupt these essential physiologic processes.

\section{Materials and Methods}

\section{Cell culture}

Cell lines were available from ATCC (T98G, MDA-MB-468, MDA-MB-231, MCF7), Clare Hall Laboratories (HCT116, SCC25, U87), or were a kind gift from Prof. Walter Bodmer (Weatherall institute of Molecular Medicine, Oxford, United Kingdom; DLD-1, Ls174T, and SW480). Cell line authentication was carried out by STR analyses (LGC Standards) 6 months prior to the first submission of the manuscript. Cells were maintained in a humidified incubator at $5 \% \mathrm{CO}_{2}$ and $37^{\circ} \mathrm{C}$. For hypoxic exposure, cells were grown in a humidified atmosphere of $0.1 \% \mathrm{O}_{2}, 5 \% \mathrm{CO}_{2}$ at $37^{\circ} \mathrm{C}$. All cell lines were maintained in DMEM supplemented with 10\% FBS except HCT116 (McCoy 5A supplemented with 10\% FBS) and SCC25 (DMEM:Ham F12 supplemented with $400 \mathrm{ng} / \mathrm{mL}$ hydrocortisone and 10\% FBS). CyQUANT (Invitrogen) was used to determine differences in viable cell counts. For spheroid culture, aggregation was initiated by plating 1,0005,000 cells into ultra-low-adherent round-bottom 96-well plates $(V W R)$ and centrifuging these at 2,000 $\times g$. Size-matched spheroids for IHC were produced by seeding different number of cells and thus preparing different spheroid sizes and treating for the same amount of time. Media at $\mathrm{pH} 7.4$ and 6.4 were prepared as described previously (26).

\section{Chemicals}

S0859 was produced by Peter Seden (University of Oxford, Oxford, United Kingdom) as described previously (30) or from Sigma-Aldrich.

\section{siRNA transfection}

Cells were transfected with HiPerFect reagent (Qiagen) according to manufacturer's instructions. Nontargeting controls were purchased from Dharmacon (D-001210-02-05) and Qiagen (1027281). siRNA oligonucleotide pools containing three sequences targeting HIF-1 $\alpha$ (5'-CAAGCAACTGTCATATATA-3' ${ }^{\prime}$, ${ }^{\prime}$-TGCCACCACTGATGAATTA- ${ }^{\prime}$, 5' ${ }^{\prime}$-TGACTCAGCTATTCACCAA- $\left.3^{\prime}\right)$ or HIF $2 \alpha$ ( $5^{\prime}$-TAACGACCTGAAGATTGAA- $3^{\prime}, 5^{\prime}$ CAAGCCACTGAGCGCAAAT- $3^{\prime}, 5^{\prime}$-TGAATTCTACCATGCGCTA-3 $3^{\prime}$ ) were purchased from Eurogentec.

\section{Stable transduction}

To knockdown SLC4A4 or SLC4A9 in Ls174T and MDA-MB231, shRNA lentivirus was purchased from Sigma-Aldrich. To knockdown SLC4A9 inducibly in U87 cells, the shRNA sequence 
against SLC4A9 (CCGGGCTGAACTTGACCCATACCTACTCGAGTAGGTATGGGTCAAGTTCAGCTTTTTG) was cloned into TetpLKO-neo (Addgene plasmid 21916; ref. 32). The CA9 knockdown in U87 is described previously (19). Lentivirus was produced using the trans-lenti shRNA packaging kit (TLP4615), and cells transduced according to the manufacturer's instructions (Thermo Scientific). Cells were grown under selective pressure; Ls174T $0.6 \mathrm{mg} / \mathrm{mL}$ G418 (Invitrogen); U87, $0.5 \mathrm{mg} / \mathrm{mL} \mathrm{G418}$ (Invitrogen), and $1 \mu \mathrm{g} / \mathrm{mL}$ puromycin (Invitrogen); and MDAMB-231, $2 \mu \mathrm{g} / \mathrm{mL}$ puromycin (Invitrogen) until no untransfected cells remained. Pools of transfected cells were used in the Ls174T and MDA-MB-231 experiments. To increase the knockdown efficiency and doxycycline control of U87 knockdown clones derived from single cells were selected for these isogenic investigations.

\section{Immunoblotting}

Cell lysates were separated on 10\% SDS-PAGE and transferred to PVDF membrane. Primary antibodies were used at 1:1,000. Rabbit anti-SLC4A9 (Sigma) anti-HIF1 $\alpha$ (BD Biosciences), antiHIF2 $\alpha$ (Cell Signaling Technology) and actin-HRP (Sigma). Appropriate secondary horseradish peroxidase-linked antibodies were used (Dako). Immunoreactivity was detected with chemiluminescence (Amersham).

\section{Quantitative PCR}

RNA extraction and the quantitative PCR protocol have been described previously (19). Data were normalized to expression of the three control genes ACTB, RPL11, and HRPT1. Primer sequences were: CA9 forward: CTTGGAAGAAATCGCTGAGG; CA9 reverse: TGGAAGTAGCGGCTGAAGTC; ACTB forward: ATTGGCAATGAGCGGTTC; ACTB reverse: GGATGCCACAGGACTCCAT; RPL11 forward: CTTTGGCATCCGGAGAAAT; RPL11 reverse: TCCAAGATTTCTTCTGCCTTG; HPRT1 forward: CCAGTCAACAGGGGACATAAA; HPRT1 reverse: CACAATCAAGACATTCTTTCCAGT. Assay on demand were purchased from Applied Biosystems for SLC4A1 (Hs00978603_m1), SLC4A2 (Hs01586776_m1), SLC4A3 (Hs00192595_m1), SLC4A4 (Hs00186798_m1), SLC4A5 (Hs00253626_m1), SLC4A7 (Hs00186192_m1), SLC4A8 (Hs00191516_m1), SLC4A9 (Hs00324675_m1), SLC4A10 (Hs00222849_m1), SLC4A11 (Hs00230695_m1), SLC26A3 (Hs00230798_m1), SLC26A4 (Hs01070620_m1), SLC26A6 (Hs00370470_m1), SLC26A7 (Hs01104163_m1), SLC26A9 (Hs00369451_m1), and ACTB (Hs99999903_m1).

\section{Measurement of intracellular $\mathrm{pH}$ in spheroids}

This was carried out as described previously (12).

\section{Xenograft studies}

Procedures were carried out under a Home Office license. Cells were trypsinized, neutralized, and washed twice in serumfree medium prior to inoculation in mice. Female 6- to 7-weekold, 16-18 g, BALB/c SCID mice (CB17/IcrHsd-Prkdc ${ }^{\text {scid }}$, Harlan) were injected orthotopically in the mammary fat pad with $25-\mu \mathrm{L}$ Matrigel (BD Biosciences) and $2.5 \times 10^{6}$ cells suspended in $25-\mu \mathrm{L}$ of serum-free medium or subcutaneously in the lower flank with 100- $\mu \mathrm{L}$ Matrigel (BD Biosciences) and $1 \times 10^{7}$ cells suspended in $100-\mu \mathrm{L}$ of serum-free medium. Tumor growth was monitored three times per week measuring the length $(L)$, width $(W)$, and height $(H)$ of each tumor using calipers. Volumes were calculated from the formula
$1 / 6 \times \pi \times L \times W \times H$. When tumors reached $1.44 \mathrm{~cm}^{3}$, mice were sacrificed by cervical dislocation. Ninety minutes prior to sacrifice, mice were injected intraperitoneally with $2 \mathrm{mg}$ of pimonidazole (Hypoxyprobe-1; Chemicon International).

\section{Immunohistochemistry}

IHC was carried out as described previously (19). Cleaved caspase-3 (R\&D Systems), Ki67 (Dako), or CA9 (M75) was incubated for 1 hour. Slides were incubated with the antirabbit secondary antibody (Dako) for 30 minutes. DAB (Dako) was applied to the sections for 7 minutes. The slides were counterstained with hematoxylin solution (Sigma-Aldrich) and mounted with Aquamount (VWR). Slides were analyzed quantitatively by image analysis in ImageJ as described previously (19)

\section{Statistical analysis}

Statistical analysis including unpaired Student $t$ test, one-way ANOVA, and linear regression of log-transformed growth data were carried out as appropriate using GraphPad Prism 4.0b.

\section{Results}

The expression of bicarbonate transporters is increased in hypoxia

The expression of the bicarbonate transporters of the SLC4 and SLC26 family members in normoxia and hypoxia $\left(0.1 \% \mathrm{O}_{2}\right.$ at 72 hours) was investigated by qRT-PCR. The expression of these genes was investigated in 10 cell lines derived from colon cancer (Ls174T, HCT116, SW480, and DLD-1), breast cancer (MDA-MB231, MDA-MB-468, and MCF7), head and neck cancer (SCC25), and glioblastoma (U87 and T98G; Fig. 1). This revealed 38 cases of significantly increased expression of the genes encoding bicarbonate transporters in response to hypoxia, and only 4 cases of significantly decreased expression (Fig. 1). Two cell lines (T98G and DLD-1) did not show significantly increased expression of any of the bicarbonate transporters (Fig. 1J and Supplementary Fig. S1I and S1J).

The pattern of hypoxia-induced expression varied between the cell lines and there did not appear to be any tissue-specific pattern, in this limited panel of cell lines. The bicarbonate transporters with the highest and most frequently observed hypoxic induction were SLC4A4 (3/10 lines), SLC4A9 (5/10), and SLC4A5 (5/10). SLC4A4 was upregulated $>100$-fold in Ls174T $(P<0.01, n=3)$, $>3$-fold in HCT116 $(P<0.001$, $n=3)$, and $>7$-fold in SCC25 $(P<0.01, n=3)$. SLC4A9 was increased $>20$-fold in Ls174T $(P<0.01, n=3)$, $>7$-fold in MDAMB-231 $(P<0.01, n=3)$, $>3$-fold in MCF7 $(P<0.05, n=3)$, and $>10$-fold in U87 $(P<0.01, n=3$; Fig. 1$)$.

Expression levels of the bicarbonate transporters for each cell line were calculated relative to $\beta$-actin (Supplementary Fig. S1). This highlights the heterogeneous pattern of bicarbonate transporter expression in each of the cell lines studied, as expected from RNA array data presented previously (28). The timing of hypoxic induction of bicarbonate transporters was investigated at 24, 48, 72, and 96 hours for all those upregulated in hypoxia for Ls174T and U87 (Supplementary Figs. S2 and S3). In the case of U87 cells, all changes identified at 72 hours were also observed at the other time points. For Ls174T, SLC4A4 was induced at all time points, whereas SLC4A3, SLC4A5, SLC4A8, SLC4A9, and SLC26A4 were 
A

\begin{tabular}{l||c|c} 
& Sodium driven & Sodium independent \\
\hline \hline \multirow{4}{*}{ Acid loaders } & & SLC4A1, SLC4A2, \\
& & SLC4A3, SLC26A3, \\
& & $\begin{array}{c}\text { SLC26A4, SLC26A7, } \\
\text { SLC26A9 }\end{array}$ \\
\hline \begin{tabular}{l|} 
Acid extruders \\
Variable or \\
unclear
\end{tabular} & SLC4A4, SLC4A6, & \\
& SLC4A7, SLC4A8, & \\
SLC4A9, SLC4A10 & SLC4A5, SLC4A11 & $\begin{array}{c}\text { SLC26A6, SLC26A8, } \\
\text { SLC26A11 }\end{array}$
\end{tabular}

C

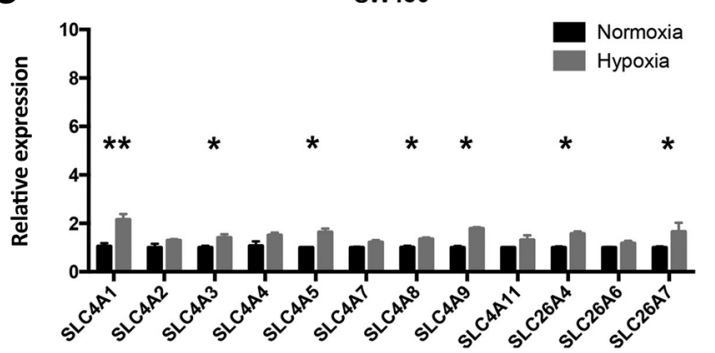

E

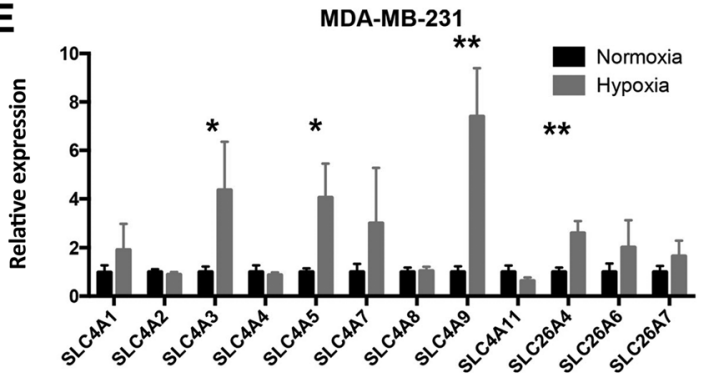

G

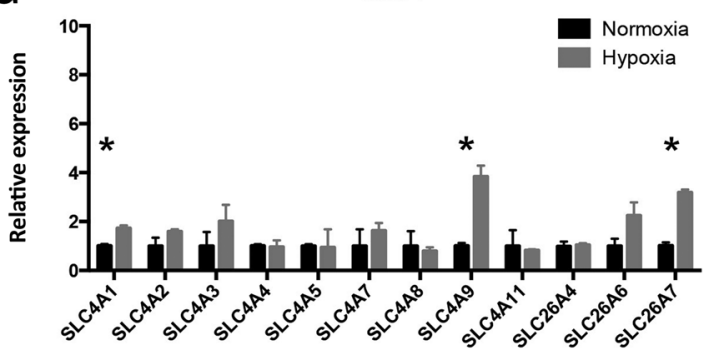

I

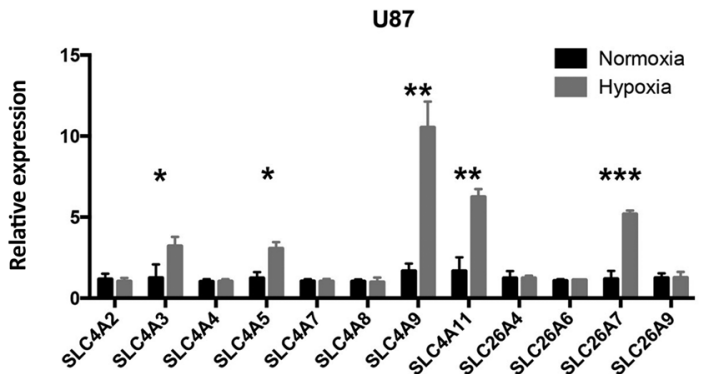

B

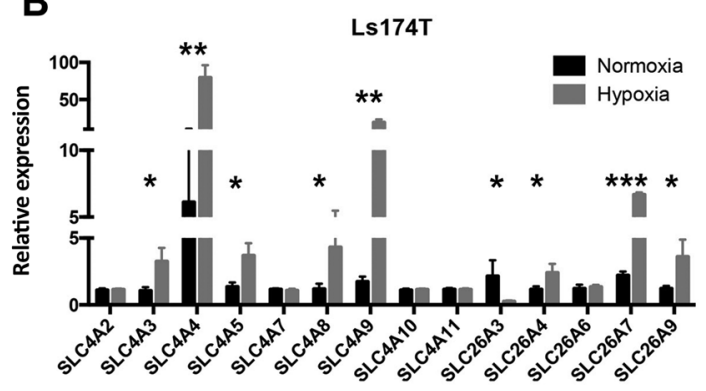

D

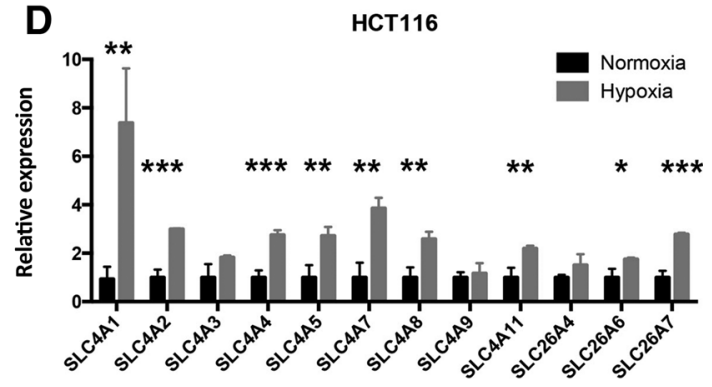

$\mathbf{F}$

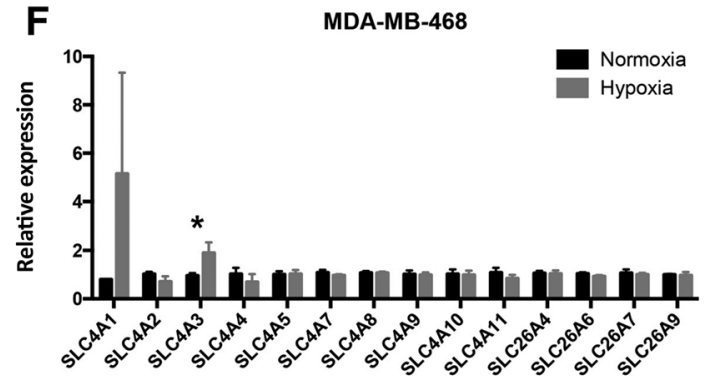

H

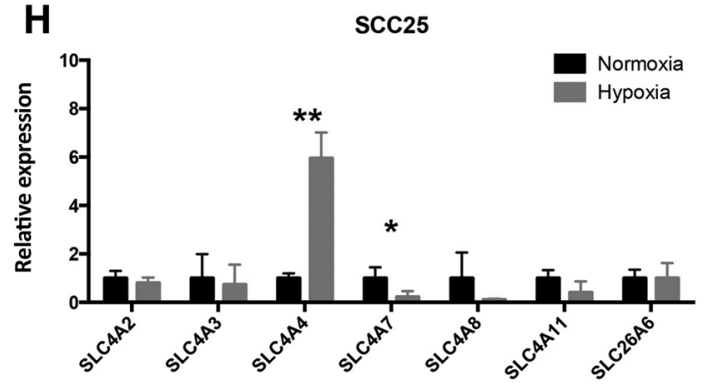

J

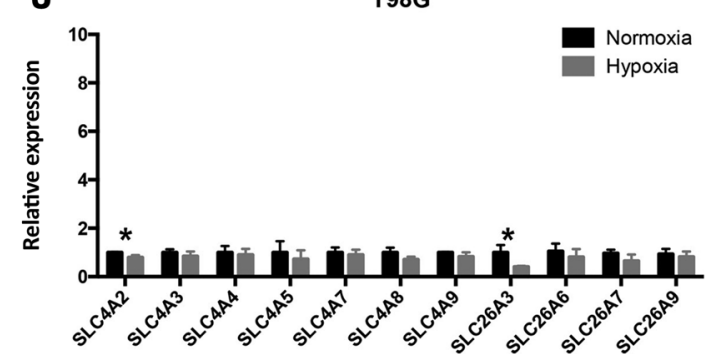

Figure 1.

The expression of bicarbonate transporters is increased in hypoxia. A, a table summarizing the functional role and $\mathrm{Na}^{+}$dependence of bicarbonate transporters of the SLC4 and SLC26 families. B-J, the relative expression of bicarbonate transporters in normoxia (black bars) and hypoxia ( $0.1 \% \mathrm{O}_{2}, 72$ hours; gray bars) in colorectal cancer cell lines (B-D), breast cancer cell lines (E-G), head and neck cancer cell line (H), and glioblastoma cell lines ( $\mathrm{I}$ and $\mathrm{J}$ ). Error bars, SD. ${ }^{* *}, P<0.001 ;{ }^{* *}, P<0.01 ;{ }^{*}, P<0.05 ; n=3$. 
A

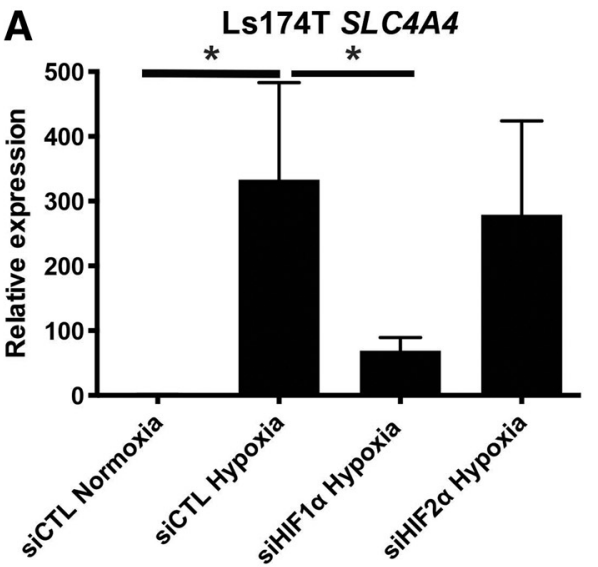

C

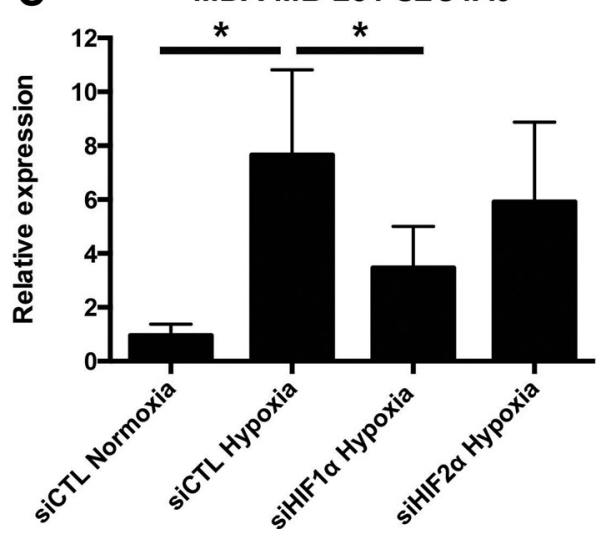

B

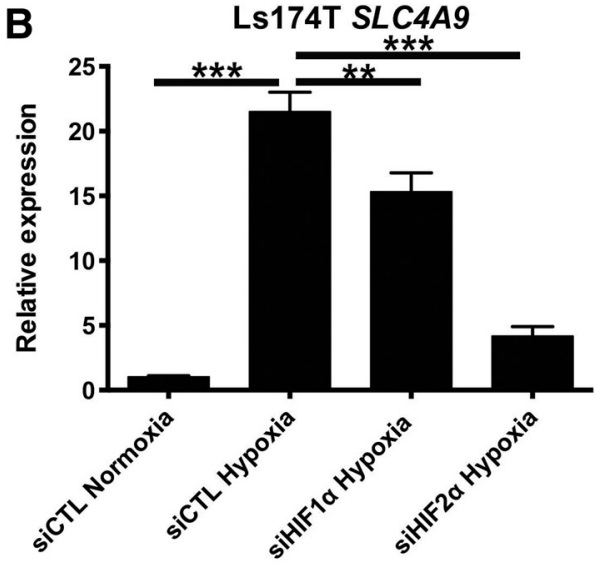

D

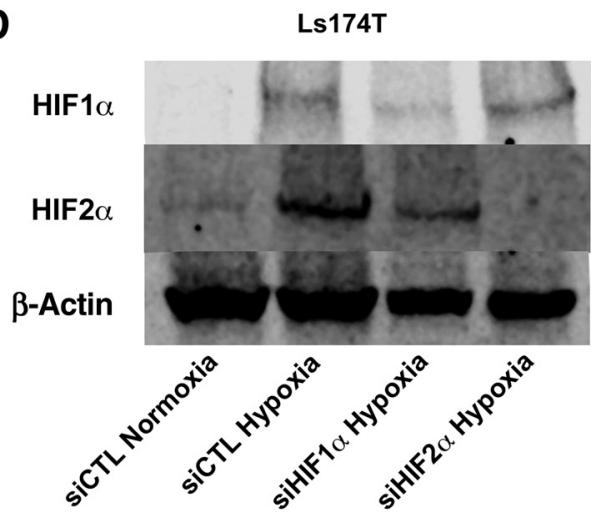

Figure 2.

Hypoxic induction of SLC4A4 and $S L C 4 A 9$ is regulated by HIF. The relative expression of SLC4A4 (A) and SLC4A9 ( $\mathrm{B}$ and $\mathrm{C}$ ) in normoxia and hypoxia $\left(0.1 \% \mathrm{O}_{2}, 72\right.$ hours) after cells were transfected with control (siCTL), HIFl $\alpha$ ( $\operatorname{siHIF1} \alpha$ ), or HIF2 $\alpha$ (siHIF2 $\alpha$ ) siRNA. D, protein validation of siHIF1 $\alpha$ and siHIF2 $\alpha$ in Ls174T in hypoxia $\left(0.1 \% \mathrm{O}_{2}\right.$ 72 hours). Error bars, SD. ${ }^{* * *}, P<0.001$; ${ }^{*}, P<0.01 ;{ }^{*}, P<0.05 ; n=3$. upregulated at 48-72 hours. The most frequently upregulated genes SLC4A4, SLC4A5, and SLC4A9 were also investigated at $1 \% \mathrm{O}_{2}$ in Ls174T, U87, and MDA-MB-231 (Supplementary Fig. S4). SLC4A4 was significantly upregulated at $1 \% \mathrm{O}_{2}$, but increases in SLC4A5 and SLC4A9 were not significant.

Hypoxic upregulation of SLC4A4 and SLC4A9 is mediated by HIF1 $\alpha$ and HIF2 $\alpha$

HIF $1 \alpha$ and HIF $2 \alpha$ were knocked down by siRNA pools and the expression of SLC4A4 and SLC4A9 was analyzed by qRTPCR. The increase in SLC4A4 expression induced by hypoxia was ablated by HIF1 $\alpha$ knockdown in $\operatorname{Ls} 174 \mathrm{~T}(P<0.05, n=3$; Fig. 2A). Hypoxic induction of SLC4A9 expression was significantly reduced by HIF1 $\alpha(P<0.01, n=3)$ or HIF $2 \alpha(P<0.001$, $n=3$ ) knockdown in Ls174T with HIF2 $\alpha$ knockdown having a greater effect on SLC4A9 hypoxic inducibility in Ls174T ( $>75 \%$, compared with $<25 \%$ with HIF1 $\alpha$ knockdown; Fig. 2B). In MDA-MB-231, HIF1 $\alpha$ knockdown significantly reduced hypoxic induction of SLC4A9 $(P<0.05, n=3$; Fig. 2C) but no significant effect was observed with HIF $2 \alpha$ knockdown. The siRNA knockdown was validated by qRT-PCR quantification and immunoblot of the expression of both $H I F 1 \alpha$ and HIF $2 \alpha$ and also by measuring CA9 RNA levels, which has been previously shown to be dependent on HIF1 $\alpha$ but not HIF2 $\alpha$ (Fig. 2D and Supplementary Fig. S5 and previously published; ref. 33). HIF-binding site sequences
(RCGTG) are found in the promoter regions of both SLC4A4 $(-786,-1690)$ and SLC4A9 $(-692,-1865,-1921)$.

\section{SLC4A4 and SLC4A9 knockdown reduces spheroid} growth rate

SLC4A4 was knocked-down in Ls174T with two different shRNAs, which significantly reduced expression of SLC4A4 in response to hypoxia $(P<0.001, n=3$; Fig. 3A). SLC4A9 protein levels were increased by hypoxia in Ls174T, MDA-MB-231, and U87 (Fig. 3B, E, and H). SLC4A9-targeting shRNA significantly reduced hypoxic induction of SLC4A9 expression at both the mRNA and protein levels (Fig. 3A, B, D, E, G, and H), in Ls174T, MDA-MB-231, and U87, compared with controls. A full representative SLC4A9 blot is shown in Supplementary Fig. S6 (MDAMB-231).

SLC4A4 knockdown reduced Ls174T spheroid growth rate by $30 \%$ compared with shCTL controls $(P<0.001, n=4$; Fig. 3C). SLC4A9 knockdown reduced spheroid growth rate compared with controls in Ls174T (30\% reduction, $P<0.001$, $n=4)$, MDA-MB-231 (39\% reduction, $P<0.05, n=3)$ and U87 (60\% reduction, $P<0.001, n=3$; Fig. $3 \mathrm{C}$, F, and I). In U87 stable doxycycline-inducible shRNAs were used to knockdown SLC4A9, followed by clone selection. The clone with the best knockdown also had a higher level of SLC4A9 expression in hypoxia than the control cells (Fig. 3G; $P<$ $0.001, n=3$ ). In $3 \mathrm{D}$ culture, the shSLC4A9 cells without 

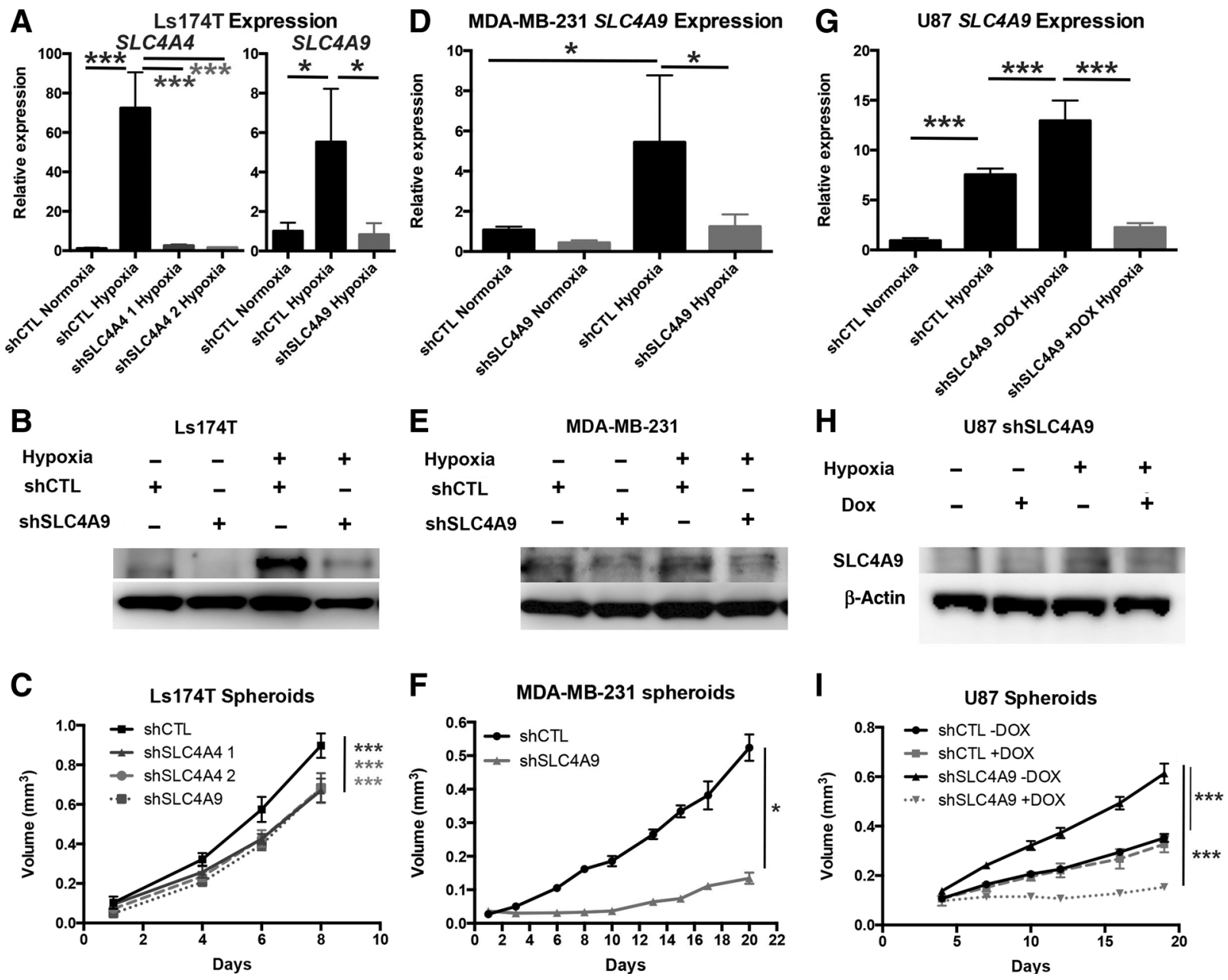

Figure 3.

SLC4A4 and SLC4A9 knockdown reduce spheroid growth rate. A, D, and G, the expression of SLC4A4 and SLC4A9 in control (shCTL) and shRNA knockdown (shSLC4A4 1 and 2 in Ls174T and shSLC4A9 in Ls174T, MDA-MB-231, and U87, respectively) at 72 hours normoxia or hypoxia $(0.1 \%$ oxygen). B, E, and H, the representative Western blots of SLC4A9 knockdown in control (shCTL) and shSLC4A9 knockdown cells (Ls174T,

MDA-MB-231, and U87, respectively) at 72 hours normoxia or hypoxia (0.1\% oxygen). C, F, and I, representative graphs of the effect of SLC4A4 or SLC4A9 knockdown on spheroid growth in Ls174T, MDA-MB-231, and U87, respectively. U87 have doxycycline (DOX)-inducible SLC4A9 knockdown. Error bars, SD. ${ }^{* * *}, P<0.001 ;{ }^{*}, P<0.05 ; n=3$.

doxycycline grew significantly faster as spheroids than the shCTL cells with or without doxycycline induction of the shRNA $(P<0.001, n=3)$.

Knockdown of SLC4A4 or SLC4A9 does not reduce cell growth in normoxia or hypoxia in 2D culture

In 2D culture, knockdown of SLC4A4 or SLC4A9 did not reduce the number of viable cells in Ls174T, MDA-MB-231, or U87 (Supplementary Fig. S6A-S6C) in normoxia or hypoxia at either pH 7.4 or 6.4 (26)

Inhibition of sodium-driven bicarbonate transporters by S0859 reduces spheroid growth rate

S0859 $(100 \mu \mathrm{mol} / \mathrm{L})$ reduced spheroid growth in Ls174T (13\%, $P<0.05, n=3)$, MDA-MB-231 (48\%, $P<0.01, n=3)$, and U87 (37\%, $P<0.05, n=3$ ) cell lines (Fig. 4A-C). The results for $\mathrm{S} 0859$ treatment of MDA-MB-231 are truncated at day 11 as these spheroids consistently disintegrated at day 12 . As shown previously (19), doxycycline-inducible CA9 knockdown reduced U87 spheroid growth rate $(37 \%, P<0.05, n=3)$. S0859 treatment in combination with CA9 knockdown further reduced spheroid growth in U87 $(70 \%, P<0.001, n=3$; Fig. 4D). 50859 treatment did not reduce the growth rate of DLD-1 (a cell line that lacked hypoxic induction of bicarbonate transporters) spheroids; however, S0859 did affect the gross appearance of DLD-1 spheroids (Supplementary Fig. S7).

Sodium-driven bicarbonate transport regulates intracellular $\mathrm{pH}$ in $3 \mathrm{D}$ spheroids

SLC4A9 knockdown resulted in more acidic steady-state intracellular $\mathrm{pH}$ in MDA-MB-231 spheroids $(P<0.05, n=$ 10 at the periphery and $P<0.01, n=10$ at the core of spheroids, $\mathrm{pH}$ difference -0.08 at the periphery and -0.14 at the core; Fig. 5A). S0859 $(100 \mu \mathrm{mol} / \mathrm{L})$ treatment resulted in lower 
A

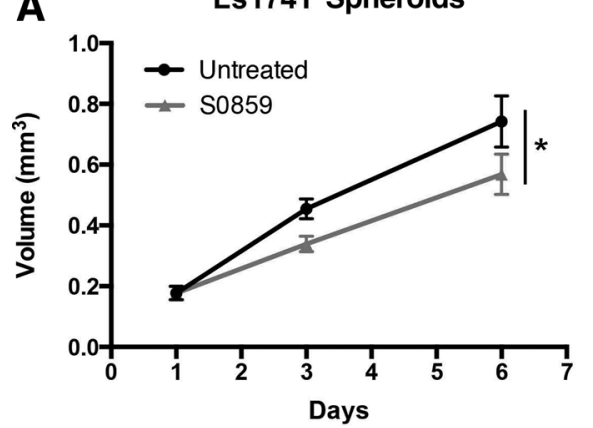

C

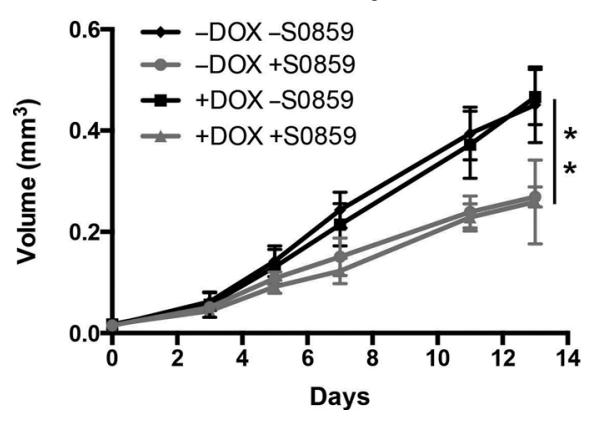

B MDA-MB-231 Spheroids

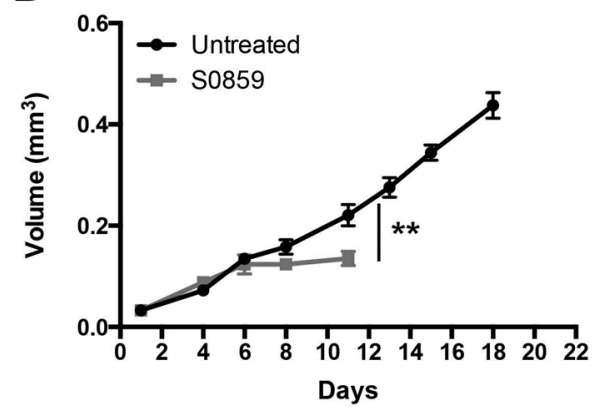

D

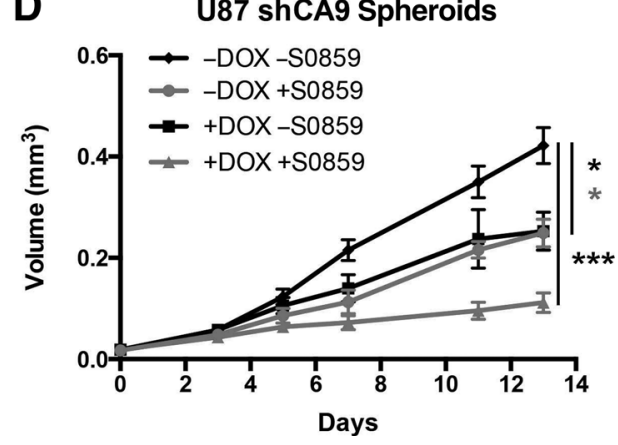

Figure 4.

Disruption of NDBTs reduces spheroid growth rate. Representative graphs of the effect of the inhibition of NDBT by S0859 on spheroid growth of Ls174T (A), MDA-MB-231 (B), U87 controls [doxycycline (DOX)-inducible shCTL; C], and U87 with doxycycline-inducible CA9 knockdown (shCA9; D). Error bars, SD. ${ }^{* *}, P<0.001 ;{ }^{* *}, P<0.01$; ${ }^{*}, P<0.05 ; n=3$. steady-state intracellular $\mathrm{pH}$ in spheroids of MDA-MB-231 $(P<$ $0.01, n=10$ at the periphery and $P<0.001, n=10$ at the core of spheroids, $\mathrm{pH}$ difference -0.11 at the periphery and -0.19 at the core; Fig. 5A) and U87 $(P<0.001, n=20$ at the periphery and the core of spheroids, $\mathrm{pH}$ difference -0.08 at the periphery and -0.29 at the core; Fig. 5B). In MDA-MB-231 spheroids, either SLC4A9 knockdown or S0859 treatment resulted in a steeper radial gradient of intracellular $\mathrm{pH}$ across the spheroid (S0859; $P<0.05, n=10$ and shSLC4A9; $P<0.05, n=10$ ) (Supplementary Fig. S8).

In U87 spheroids, the effect of S0859 on intracellular pH was greatest at the periphery rather than the core (Fig. 5B). To explore the reasons for this effect, size-matched U87 spheroids were immunostained for HIF1 $\alpha$, CA9, and Ki67 (a marker for proliferation; Fig. 5D). This revealed that HIF $1 \alpha$ is stabilized throughout most of the U87 spheroid, except a single-cell rim at the periphery. Accordingly, the pattern of CA9 expression was similar to that of HIF $1 \alpha$. Ki67 positivity was greater at the periphery of spheroids compared with the core (Fig. 5C). Investigation of the impact of $\mathrm{S} 0859$ on the Ki67 positivity (Supplementary Fig. S7) showed a significant reduction (from $24 \%$ to $2 \% P<0.001)$ for Ki67 positivity. The reliance on CA9 and bicarbonate transport for $\mathrm{pH}$ control is likely to be greatest where the cells are undergoing active proliferation and produce greater quantities of metabolic acid. In U87 spheroids, SLC4A9 knockdown had a similar effect on intracellular $\mathrm{pH}$ as with MDA-MB-231 spheroids, where the greatest effect was observed at the core of the spheroid $\left(\mathrm{P}<0.01, \Delta \mathrm{pH}=\mathrm{pH}_{\text {periphery }}-\mathrm{pH}_{\text {core }}=\right.$ -0.09 at the periphery and $P<0.001, \Delta \mathrm{pH}=-0.22$ at the core of spheroids; Fig. 5C). Measurements of the dynamics of intracellular $\mathrm{pH}$ recovery demonstrated that SLC4A9 is a key acid-extruding protein, particularly important at the core of the spheroid $(P<0.001, \Delta \mathrm{pH}=-0.20 ; P<0.05, \Delta \mathrm{pH}=$ -0.04 ; Fig. 5E and F).
Knockdown or inhibition of sodium-driven bicarbonate transporters increases apoptosis in the core of spheroids NDBT knockdown or S0859-treated spheroids were stained for cleaved caspase-3 (a marker for apoptosis). SLC4A4 knockdown did not increase apoptosis in Ls174T (Fig. 6A). In contrast, SLC4A9 knockdown increased apoptosis in MDAMB-231 $(P<0.01, n=3)$ but not in Ls174T or U87 (Fig. 6A-C). S0859 increased apoptosis in Ls174T $(P<0.01$, $n=3)$, MDA-MB-231 $(P<0.001, n=3)$, and U87 $(P<0.001$, $n=3$; Fig. 6D-F).

SLC4A9 knockdown reduces tumor growth rate in vivo

Doxycycline-inducible SLC4A9 knockdown reduced the growth of $U 87$ xenografts by $92 \%(P<0.0001, n=7$; Fig. 7$)$. There was no effect of doxycycline on U87 shCTL control xenograft growth rates (Supplementary Fig. S8). SLC4A9 knockdown reduced the growth rate of MDA-MB-231 orthotopic xenografts by 79\% $(P<0.0001, n=6$; Fig. 7$)$.

\section{Discussion}

The increased acidity associated with the hypoxic tumor microenvironment places additional stress on cells already under metabolic adaptation to low oxygen. HIF increases the expression of many $\mathrm{pH}$-regulating proteins (34-36). Cells within the hypoxic microenvironment may then become sensitive to inhibition or knockdown of pH-regulatory proteins resulting in induced essentiality or context-dependent lethality (37).

Here, we identify increased expression of several bicarbonate transporters in response to hypoxia across a panel of cell lines from four different cancer types. $0.1 \% \mathrm{O}_{2}$ was used in these investigations as the physiologically relevant oxygen partial pressure for tumors, based on in vivo oxygen electrode measurements. 
A

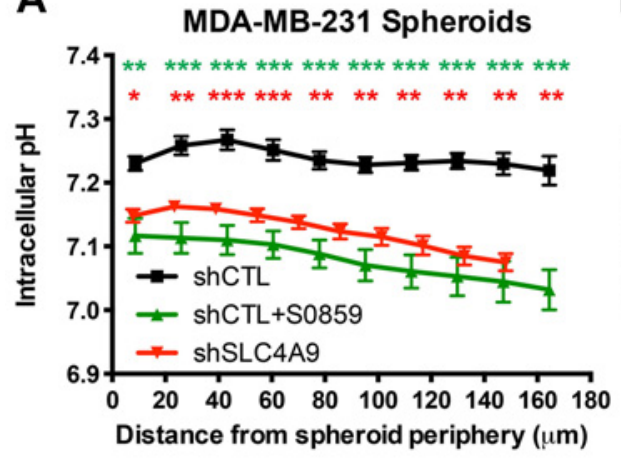

NDBTs regulate intracellular $\mathrm{pH}$ in 3D spheroids. Measurements of the intracellular $\mathrm{pH}$ of MDA-MB-231 (A) and U87 spheroids ( $B, C, E$, and $F$ ). The intracellular $\mathrm{pH}$ is averaged in 10 concentric rings in the spheroid and data points for each concentric ring is shown in the middle of each ring. Effect of treatment with S0859 $(100 \mu \mathrm{mol} / \mathrm{L})$ for 1 hour on MDA-MB-231 (A) and U87 spheroids (B) and SLC4A9 knockdown on MDA-MB-231 (A) and U87 spheroids (C, E, and F). A-C, resting $\mathrm{pH}$ measurements. $E$ and $F$, recovery of intracellular $\mathrm{pH}$ after ammonium prepulse. C, representative images of staining for CA9, HIFl $\alpha$, and Ki67 (dark brown staining denotes positivity) of U87 spheroids. Scale bars, $200 \mu \mathrm{m}$. Error bars, SE. ${ }^{* * *}, P<0.001 ;{ }^{* *}, P<0.01$; $P<0.05 ; n=20$ (MDA-MB-231); $n=10-15$ (U87)

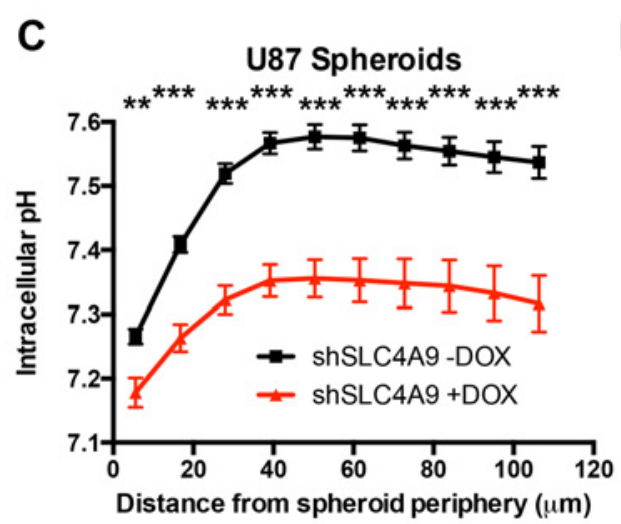

B

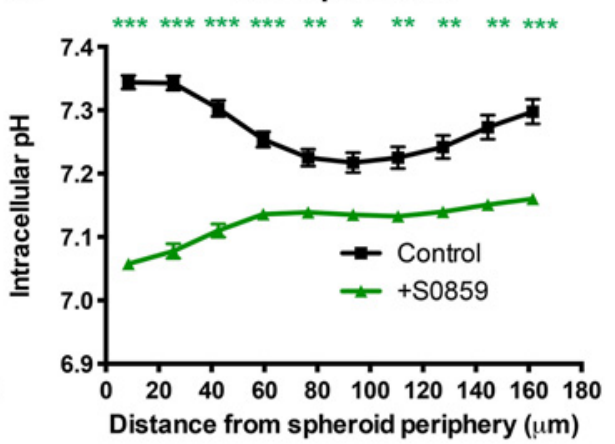

D

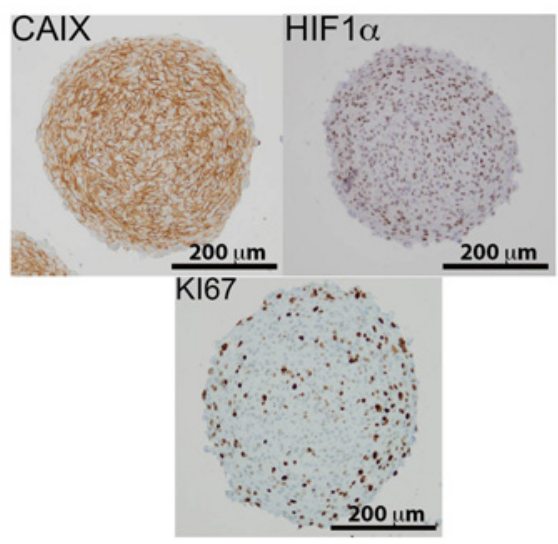

$\mathbf{F}$

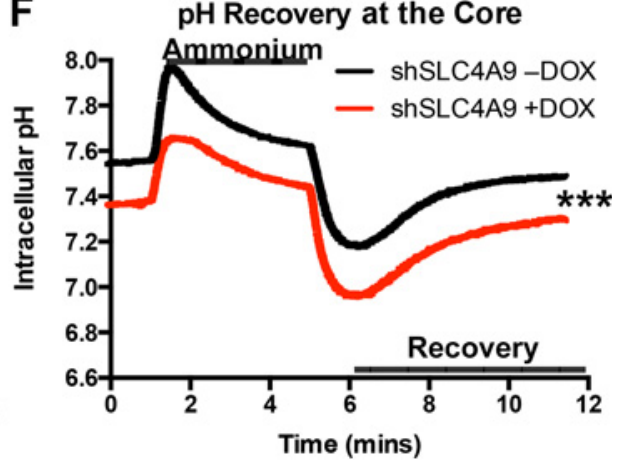

For example, approximately $25 \%$ of head and neck, breast, and uterine cervix tumors have a $\mathrm{pO}_{2}$ of $\leq 2.5 \mathrm{mmHg}$ equivalent to $0.32 \%$ oxygen (38). Investigations at $1 \%$ oxygen identified fewer statistically significant changes. We tested whether the hypoxic induction of SLC4A4 and SLC4A9 was dependent on HIF1 $\alpha$ or HIF2 $\alpha$ (Fig. 2). SLC4A9 was most profoundly regulated by HIF $2 \alpha$ in Ls174T and by HIF1 $\alpha$ in MDA-MB-231. Similar examples from different cell lines of other genes that are regulated by alternate HIF proteins have been identified previously. For example, VEGF is primarily regulated by HIF $1 \alpha$ in MCF7 cells and by HIF $2 \alpha$ in RCC4 cells (39). Indeed, the hypoxic regulation of gene expression by HIF $1 \alpha$ and HIF $2 \alpha$ is complex and dependent upon cofactors and epigenetics. This regulatory complexity may explain the heterogeneity in patterns of expression for the bicarbonate transporters. Nonetheless, we have been able to identify a general upregulation of one or more functionally related NDBTs in the majority of cell lines investigated. Further investigations would be required to identify if factors in addition to HIF are involved in the regulation of the hypoxic induction of these bicarbonate transporters. SLC4A7 had high relative levels of expression, but was only significantly upregulated in HCT116 and was downregulated in SCC25. SLC4A7 has been investigated previously and was found to be regulated by ErbB1, 2, and 3 in breast cancer cell lines (28). The significance of SLC4A7 in cancer has been reviewed in ref. 28 .

We show that knockdown or inhibition of sodium-driven bicarbonate transporters acidifies the $\mathrm{pH}$ of cells in spheroids. For SLC4A9 knockdown in MDA-MB-231 and U87 and for S0859 treatment in MDA-MB-231, this effect was greatest at the spheroid core. Similarly, measurements of intracellular $\mathrm{pH}$ recovery in U87 spheroids demonstrated clearly that SLC4A9 had a more substantial effect on pH regulatory fluxes at the core. In U87, the effect of S0859 treatment was present across the spheroid with most pronounced $\mathrm{pH}_{\mathrm{i}}$ difference at the spheroid periphery. Given the 
A

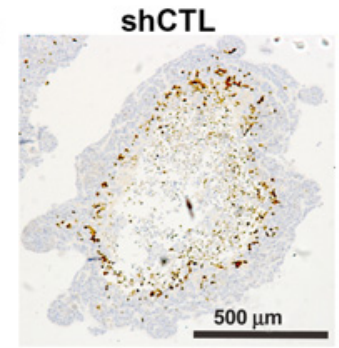

B

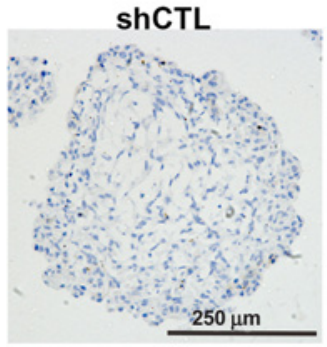

C

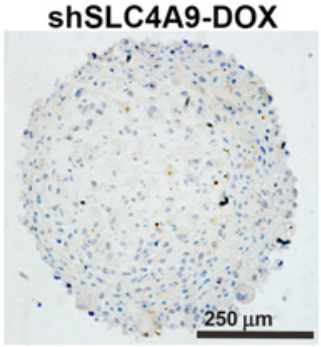

D

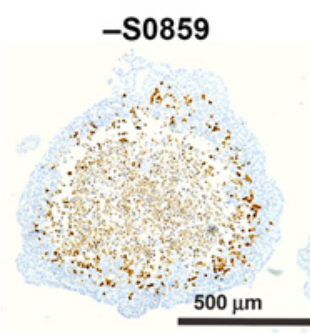

E

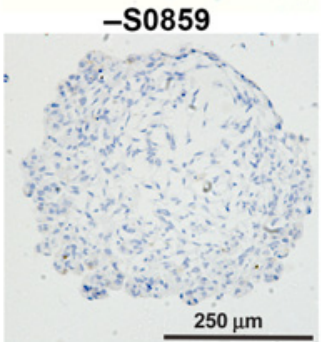

F

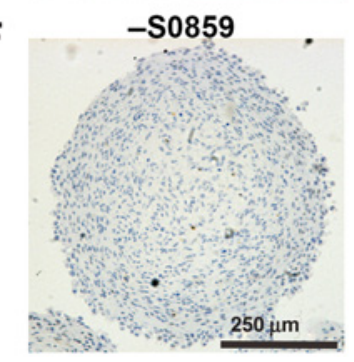

shSLC4A4 1

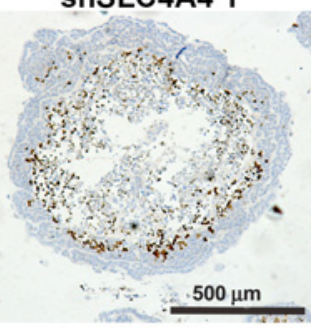

shSLC4A9

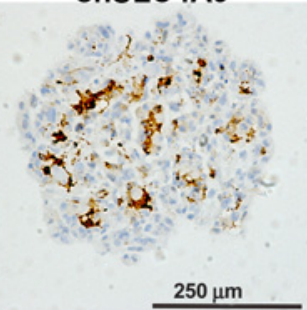

shSLC4A9+DOX

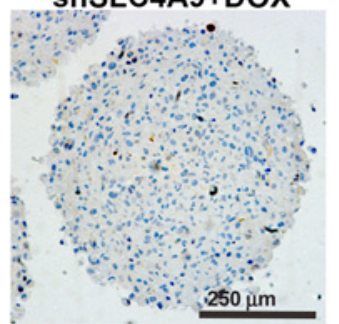

$+\mathbf{S} 0859$

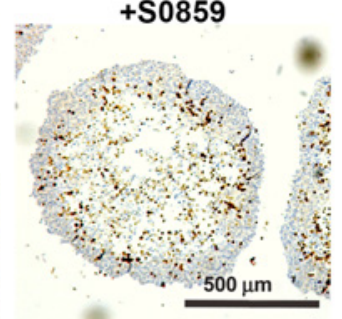

$+\mathbf{S} 0859$

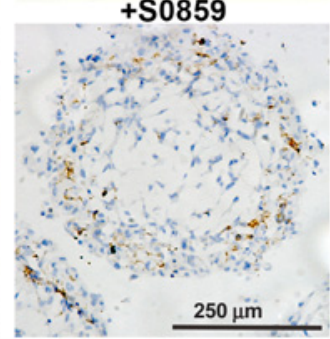

$+\mathbf{S} 0859$

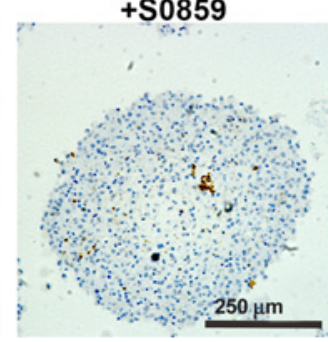

Ls174T

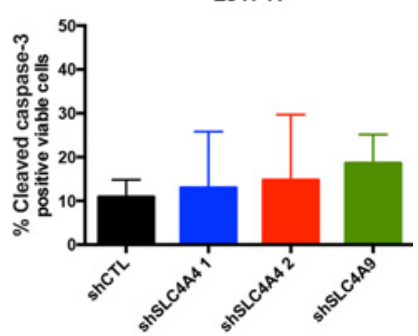

MDA-MB-231

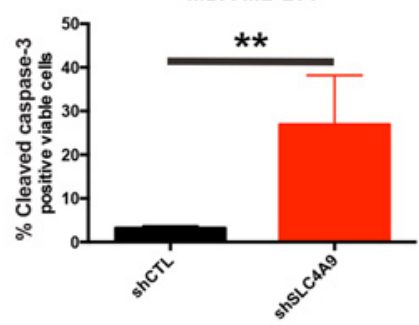

U87 ShSLC4A9
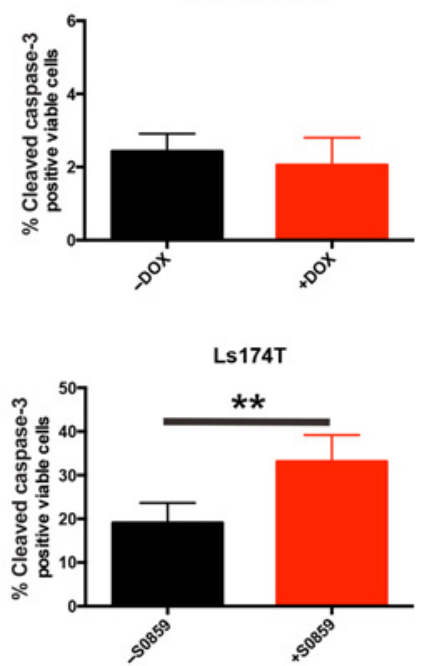

MDA-MB-231
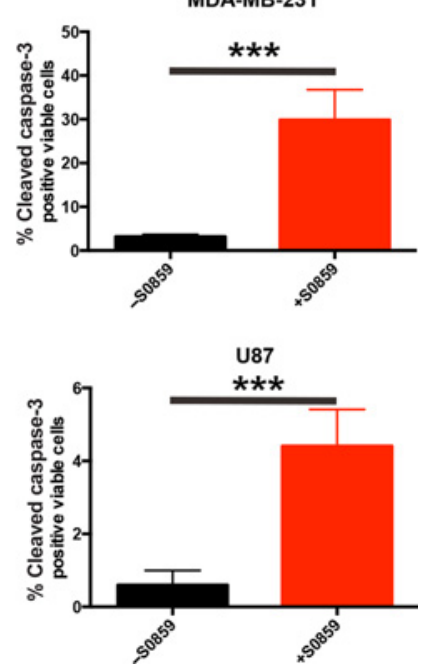

Figure 6.

Knockdown or inhibition of NDBTs increases apoptosis in the core of spheroids. SLC4A9 knockdown increased cleaved caspase-3 positivity in MDA-MB-231 but not in Ls174T or U87 (A-C). SLC4A4 knockdown did not increase apoptosis in Ls174T (A). 50859 treatment increased cleaved caspase-3 positivity in Ls174T, MDA-MB-231, and U87 (D-F). Error bars, SD; ${ }^{* * *}, P>0.001 ; * *, P<0.01$; $n=3$. 
Figure 7.

Knockdown of NDBT SLC4A9 reduces xenograft growth. SLC4A9 knockdown reduced growth of U87 (glioblastoma, $n=7 ; \mathrm{A}$ ) and MDA-MB-231 (breast cancer, $n=6 ; \mathrm{B}$ ) xenografts. Error bars, SD. ${ }^{* * * *}, P<0.0001$
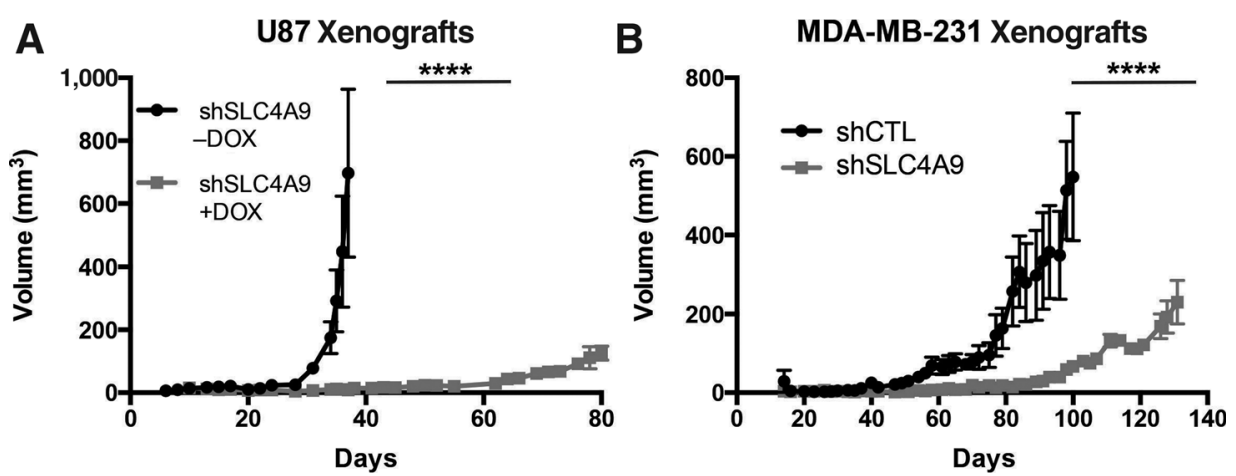

increased hypoxic expression of sodium-driven bicarbonate transporters and the exacerbated acid stress in the hypoxic microenvironment, we expected a greater effect of knockdown/inhibition at the core of spheroids. The surprising effects on $\mathrm{pH}$ in U87 spheroids can be explained in terms of greater cell proliferation, and hence metabolic acid production, near the periphery, which is already considerably hypoxic (based on HIF and CA9 staining). S0859 markedly reduces Ki67 positivity (proliferation) at the periphery of U87 spheroids, and therefore the associated metabolic acid production. An effect on the intracellular $\mathrm{pH}$ in peripheral regions would also be consistent with inhibition of a membrane transporter active throughout the spheroid, responsible for setting steady-state intracellular $\mathrm{pH}$. Thus, ablation of such an acid-extruding transporter would result in a drift toward the acidic direction of steady-state $\mathrm{pH}$ across all spheroid regions. The difference in the effects of SLC4A9 knockdown versus S0859 treatment on intracellular pH in U87 is likely due to the breadth of targeting: knockdown eliminates a specific NDBT gene product, whereas S0859 targets multiple bicarbonate transporters.

We showed that knockdown or inhibition of sodium-driven bicarbonate transport reduced spheroid growth rate, in agreement with reduced intracellular $\mathrm{pH}$, a known suppressor of proliferation $(8,19)$. Furthermore, we showed that S0859 treatment had a greater effect on spheroid growth when combined with CA9 knockdown. This suggests that combined bicarbonate transporter and CAIX targeting may provide the greatest therapeutic impact. Bicarbonate transport is important for regulating the intracellular $\mathrm{pH}$ (hence growth) not only in hypoxic but also normoxic tumor microenvironment. However, increased bicarbonate transporter expression in hypoxia would offer an advantage, particularly if coupled with a stimulated rate of metabolic acid production. However, in the case of cell lines with high normoxic (i.e., baseline) levels of bicarbonate transport, additional hypoxic induction may not yield an advantage, as for the case for DLD-1 (Supplementary Fig. S1).

SLC4A4 knockdown in Ls174T cells, and SLC4A9 knockdown in LS174T, MDA-MB-231, and U87 did not reduce the number of viable cells in $2 \mathrm{D}$ culture in normoxia or hypoxia at $\mathrm{pH} 7.4$ or 6.4 (26). This suggests that studies in $2 \mathrm{D}$ do not recapitulate the properties of 3D tissue, in which SLC4A4 and SLC4A9 clearly play a major homeostatic role. These additional properties, evident only in $3 \mathrm{D}$ tissue, may relate to stresses associated with the hypoxic microenvironment, such as metabolic stress and expanded diffusion distances.

A key result from this study is the increased apoptosis identified in spheroids with SLC4A9 knockdown in MDAMB-231 or S0859 treatment in MDA-MB-231, U87, and
Ls174T. The large increase in apoptosis at the core of MDAMB-231 spheroids is the probable cause of spheroid disintegration at day 12. In contrast, the degree of apoptosis seen with S0859 in U87 spheroids is small and unlikely to have as significant a clinical impact (regression) as the effect seen upon reducing proliferation for these cells. The absence of an increase in apoptosis in U87 and Ls174T with knockdown of a single bicarbonate transporter may be due to a compensatory effect by one or more other transporters that are also increased in hypoxia. In MDA-MB-231, we showed a similar effect with knockdown of SLC4A9 alone and treatment with S0859. Like most pharmacologic inhibitors, recent data identified possible off-target effects of 50859 including inhibition of lactate transporters ectopically expressed in oocytes (31). Given the similarities in responses between S0859 and the NDBT knockdown studies, it is likely that the NDBT are the major targets here as described previously $(7,29,30)$.

Finally, we show that SLC4A9 is important for xenograft growth in vivo, and that this effect was more substantial than in $3 \mathrm{D}$ in vitro models. This protein has not been studied in the context of cancer, and therefore is a novel target. The dependency on one type of transporter argues that these can specialize to an extent that cannot be compensated for by other proteins in these cells. Knockdown of NDBT transporters with additional shRNA sequences or reexpression of NDBT after the shRNA knockdown would provide extra validation to the approaches utilized here.

A recent publication highlighted the possible impact of doxycycline on mitochondrial gene expression (40). In our experiments, we included doxycycline-treated controls for both the in vivo (Supplementary Fig. S8B) and in vitro (Figs. 3I and 4C) studies and no effect of doxycycline alone was identified. Furthermore we have combined the treatment of U87 shCTL cells with doxycycline and the NBDT inhibitor S0859 in long-term spheroid experiments (Fig. 4C). There was no additional impact on growth when doxycycline treatment is combined with NDBT inhibition compared with NDBT inhibition alone. These data suggest that the possible mitochondrial impact of doxycycline is unlikely to be producing a combination effect in our studies. However it is possible that when doxycycline is combined with drugs/gene knockdown that reduce the rate of glycolysis the interpretation of the results would become more difficult. The NBDT inhibitor S0859 used here also inhibits other pH regulators such as MCT1 (at $10 \mu \mathrm{mol} / \mathrm{L}$ ), MCT2 (at $4 \mu \mathrm{mol} / \mathrm{L}$ ), and MCT4 (at $5 \mu \mathrm{mol} / \mathrm{L}$ ) (31) and these may also contribute to its effectiveness, although no greater effect than the knockdown of a single transporter was seen. 
Further to the effect of hypoxia on the expression of acidextruding bicarbonate transporters, acid-loading bicarbonate transporters also showed increased expression in at least one cell line. Acid-extruders generate flux in the acidic range of intracellular $\mathrm{pH}$; whereas acid-loaders are activated at high intracellular $\mathrm{pH}$. The fluxes are balanced (i.e., equal but opposite) at resting intracellular $\mathrm{pH}$. An increase in the expression (hence activity) of acid extruders will protect cells more effectively from acid challenges. However, if acid extruders alone were upregulated, steadystate intracellular $\mathrm{pH}$ would drift towards more alkaline values, which may not be permissive for cells. Thus, to bring steady state (i.e., setpoint) intracellular $\mathrm{pH}$ to normal levels, acid loaders would need to be upregulated concurrently. Thus, higher acid extrusion and acid-loading fluxes, attained by hypoxia-induction of their genes, produces a homeostatic system that protects intracellular $\mathrm{pH}$ better (faster) without shifting steady-state (set-point) intracellular $\mathrm{pH}$. The functional significance of this observed effect at expression levels merits further investigation with detailed flux analyses.

The lack of strong correlation between the expression of bicarbonate transporters and hypoxic markers (e.g., CA9; ref. 28) is consistent with the isoform-dependent hypoxic regulation of bicarbonate transporters across cell lines. Thus, there is considerable heterogeneity in how tumor cells regulate their $\mathrm{pH}$, and this will be essential to study in clinical studies, where $\mathrm{pH}$ can be measured by MRS, and NDBT and CA9 expression can be analyzed by PCR and IHC.

Given the probable utility of targeting sodium-driven bicarbonate transport in cancer, it is important to consider toxicity. Gene knockout studies in mice provide further insight into likely toxicity of systemic NDBT inhibition. SLC4A4 knockout mice have multiple abnormalities including: metabolic acidosis, growth retardation, and death before weaning (41). Human patients with homozygous SLC4A4-inactivating mutations exhibit ocular abnormalities and renal tubular acidosis $(42,43)$. Homozygous SLC4A5 mutation in mice resulted in metabolic acidosis and arterial hypertension (44). Mice lacking SLC4A7 develop blindness and auditory impairment (45). SLC4A9 knockout mice have no obvious phenotypic abnormalities (46). SLC4A10 plays a role in control of neuronal $\mathrm{pH}$ and excitability and cerebrospinal fluid secretion $(47,48)$. The majority of effects, but not all, associated with NDBT gene knockouts or homozygous deletions is developmental in pathogenesis, suggesting that targeting one or more of these may be viable. In particular, the striking effects of SLC4A9 knockdown on tumor growth in vivo and the lack of reported phenotypic abnormalities in SLC4A9 knockout mice make it an ideal candidate for specific inhibition. Alternatively, given the dependence of cells in the hypoxic microenvironment on NDBT for survival, utilizing a hypoxia-activated prodrug would enable targeting tumor cells in the hypoxic therapy-resistant microenvironment in combination treatments (49). Targeting NDBT has the advantage over targeting CA9 as inhibition or knockdown induces increased apoptosis, whereas CA9 inhibition/ knockdown decreases apoptosis (19). The mechanism here is significantly different to that proposed for buffer therapy (50). Buffer therapy increases systemic bicarbonate with the aim to neutralize extracellular acidosis (50). In contrast, blocking bicarbonate transport disrupts intracellular acid-base homeostasis. Interestingly, inhibition of cellular bicarbonate uptake may, in fact, raise extracellular buffering by transferring bicarbonate out of the acidified intracellular compartment (that, when alkaline, stores most of the tissue's bicarbonate). Effectively, this may improve buffer therapy efficacy.

In summary, we identified hypoxic regulation of tumor $\mathrm{pH}$, via sodium-driven bicarbonate transporters in a panel of cell lines across four tumor types. We showed profound effects of inhibition of SLC4A9 in vivo showing the importance of this transporter in the tumor microenvironment. Targeting sodiumdriven bicarbonate transport should be an effective useful strategy for combination with hypoxia inducing antiangiogenic therapy.

\section{Disclosure of Potential Conflicts of Interest}

P. Seden reports receiving a commercial research grant from the Cancer Research UK Small Molecule Cancer Drug Discovery Award (C17468/A9332). No potential conflicts of interest were disclosed by the other authors.

\section{Authors' Contributions}

Conception and design: A. McIntyre, S. Wigfield, P. Swietach, A.L. Harris Development of methodology: A. McIntyre, I. Ledaki, C. Snell, J.-L. Li, P. Swietach

Acquisition of data (provided animals, acquired and managed patients, provided facilities, etc.): A. McIntyre, A. Hulikova, D. Singleton, D. Jones, E. Bridges, S. Wigfield, J.-L. Li, P. Swietach

Analysis and interpretation of data (e.g., statistical analysis, biostatistics, computational analysis): A. McIntyre, A. Hulikova, I. Ledaki, E. Bridges, J.-L. Li, P. Swietach, A.L. Harris

Writing, review, and/or revision of the manuscript: A. McIntyre, A. Hulikova, I. Ledaki, C. Snell, D. Singleton, E. Bridges, P. Swietach, A.L. Harris Administrative, technical, or material support (i.e., reporting or organizing data, constructing databases): A. Hulikova, C. Snell, G. Steers, A.L. Harris Study supervision: A. McIntyre, A.L. Harris

Other (chemical synthesis of molecular probe): $P$. Seden

Other (provision of study material): A. Russell

\section{Acknowledgments}

This work was funded by grants from Cancer Research UK (A.L. Harris and A. Russell), Breast Cancer Research Foundation (A.L. Harris), Breast Cancer Now (A. McIntyre), Worldwide Cancer Research (P. Swietach), and Royal Society (P. Swietach).

The costs of publication of this article were defrayed in part by the payment of page charges. This article must therefore be hereby marked advertisement in accordance with 18 U.S.C. Section 1734 solely to indicate this fact.

Received July 13, 2015; revised April 25, 2016; accepted April 25, 2016; published OnlineFirst May 17, 2016.

\footnotetext{
References

1. Vaupel P, Okunieff P, Neuringer LJ. Blood flow, tissue oxygenation, $\mathrm{pH}$ distribution, and energy metabolism of murine mammary adenocarcinomas during growth. Adv Exp Med Biol 1989;248:835-45.

2. Rebucci M, Michiels C. Molecular aspects of cancer cell resistance to chemotherapy. Biochem Pharmacol 2013;85:1219-26.
}

3. McIntyre A, Harris AL. Metabolic and hypoxic adaptation to anti-angiogenic therapy: a target for induced essentiality. EMBO Mol Med 2015;7: 368-79.

4. Shen C, Kaelin WG Jr. The VHL/HIF axis in clear cell renal carcinoma Semin Cancer Biol 2013;23:18-25. 
5. Helmlinger G, Yuan F, Dellian M, Jain RK. Interstitial pH and pO2 gradients in solid tumors in vivo: high-resolution measurements reveal a lack of correlation. Nat Med 1997;3:177-82.

6. Swietach P, Vaughan-Jones RD, Harris AL. Regulation of tumor $\mathrm{pH}$ and the role of carbonic anhydrase 9. Cancer Metastasis Rev 2007;26:299-310.

7. Hulikova A, Vaughan-Jones RD, Swietach P. Dual role of CO2/HCO3(-) buffer in the regulation of intracellular $\mathrm{pH}$ of three-dimensional tumor growths. J Biol Chem 2011;286:13815-26.

8. Pouyssegur J, Franchi A, L'Allemain G, Paris S. Cytoplasmic pH, a key determinant of growth factor-induced DNA synthesis in quiescent fibroblasts. FEBS Lett 1985;190:115-9.

9. Rofstad EK, Mathiesen B, Kindem K, Galappathi K. Acidic extracellular pH promotes experimental metastasis of human melanoma cells in athymic nude mice. Cancer Res 2006;66:6699-707.

10. Kaluz S, Kaluzova M, Liao SY, Lerman M, Stanbridge EJ. Transcriptional control of the tumor- and hypoxia-marker carbonic anhydrase 9: a one transcription factor (HIF-1) show? Biochim Biophys Acta 2009;1795:162-72.

11. Swietach P, Wigfield S, Cobden P, Supuran CT, Harris AL, Vaughan-Jones RD. Tumor-associated carbonic anhydrase 9 spatially coordinates intracellular $\mathrm{pH}$ in three-dimensional multicellular growths. J Biol Chem 2008;283:20473-83.

12. Swietach P, Patiar S, Supuran CT, Harris AL, Vaughan-Jones RD. The role of carbonic anhydrase 9 in regulating extracellular and intracellular ph in three-dimensional tumor cell growths. J Biol Chem 2009;284:20299-310.

13. Hui EP, Chan AT, Pezzella F, Turley H, To KF, Poon TC, et al. Coexpression of hypoxia-inducible factors $1 \alpha$ and $2 \alpha$, carbonic anhydrase IX, and vascular endothelial growth factor in nasopharyngeal carcinoma and relationship to survival. Clin Cancer Res 2002;8:2595-604.

14. Chia SK, Wykoff CC, Watson PH, Han C, Leek RD, Pastorek J, et al. Prognostic significance of a novel hypoxia-regulated marker, carbonic anhydrase IX, in invasive breast carcinoma. J Clin Oncol 2001;19:3660-8.

15. Watson PH, Chia SK, Wykoff CC, Han C, Leek RD, Sly WS, et al. Carbonic anhydrase XII is a marker of good prognosis in invasive breast carcinoma. Br J Cancer 2003;88:1065-70.

16. Giatromanolaki A, Koukourakis MI, Sivridis E, Pastorek J, Wykoff CC, Gatter KC, et al. Expression of hypoxia-inducible carbonic anhydrase-9 relates to angiogenic pathways and independently to poor outcome in non-small cell lung cancer. Cancer Res 2001;61:7992-8.

17. Chiche J, Ilc K, Laferriere J, Trottier E, Dayan F, Mazure NM, et al. Hypoxiainducible carbonic anhydrase IX and XII promote tumor cell growth by counteracting acidosis through the regulation of the intracellular $\mathrm{pH}$. Cancer Res 2009;69:358-68.

18. Lou Y, McDonald PC, Oloumi A, Chia S, Ostlund C, Ahmadi A, et al. Targeting tumor hypoxia: suppression of breast tumor growth and metastasis by novel carbonic anhydrase IX inhibitors. Cancer Res 2011;71:3364-76.

19. McIntyre A, Patiar S, Wigfield S, Li JL, Ledaki I, Turley H, et al. Carbonic anhydrase IX promotes tumor growth and necrosis in vivo and inhibition enhances anti-VEGF therapy. Clin Cancer Res 2012;18:3100-11.

20. Swietach P, Hulikova A, Vaughan-Jones RD, Harris AL. New insights into the physiological role of carbonic anhydrase IX in tumour $\mathrm{pH}$ regulation. Oncogene 2010;29:6509-21.

21. Romero MF, Chen AP, Parker MD, Boron WF. The SLC4 family of bicarbonate $(\mathrm{HCO}(3)(-))$ transporters. Mol Aspects Med 2013;34:159-82.

22. Alper SL. Molecular physiology of SLC4 anion exchangers. Exp Physiol 2006;91:153-61.

23. Alper SL, Sharma AK. The SLC26 gene family of anion transporters and channels. Mol Aspects Med 2013;34:494-515.

24. Parker MD, Boron WF, Tanner MJA. Characterization of human 'AE4' as an electroneutral, sodium-dependent bicarbonate transporter. FASEB J 2002; 16:A796-A.

25. Hulikova A, Harris AL, Vaughan-Jones RD, Swietach P. Regulation of intracellular $\mathrm{pH}$ in cancer cell lines under normoxia and hypoxia. J Cell Physiol 2013;228:743-52.

26. Parks SK, Pouyssegur J. The $\mathrm{Na}(+) / \mathrm{HCO}(-)$ Co-transporter SLC4A4 plays a role in growth and migration of colon and breast cancer cells. J Cell Physiol 2015;230:1954-63.

27. Suo WH, Zhang N, Wu PP, Zhao L, Song LJ, Shen WW, et al. Anti-tumour effects of small interfering RNA targeting anion exchanger 1 in experimental gastric cancer. Br J Pharmacol 2012;165:135-47.

28. Gorbatenko A, Olesen CW, Boedtkjer E, Pedersen SF. Regulation and roles of bicarbonate transporters in cancer. Front Physiol 2014;5:130.
29. Ch'en FF, Villafuerte FC, Swietach P, Cobden PM, Vaughan-Jones RD. S0859, an N-cyanosulphonamide inhibitor of sodium-bicarbonate cotransport in the heart. Br J Pharmacol 2008;153:972-82.

30. Larsen AM, Krogsgaard-Larsen N, Lauritzen G, Olesen CW, Honore Hansen S, Boedtkjer E, et al. Gram-scale solution-phase synthesis of selective sodium bicarbonate co-transport inhibitor S0859: in vitro efficacy studies in breast cancer cells. ChemMedChem 2012;7:1808-14.

31. Heidtmann H, Ruminot I, Becker HM, Deitmer JW. Inhibition of monocarboxylate transporter by N-cyanosulphonamide S0859. Eur J Pharmacol 2015;762:344-9.

32. Wiederschain D, Wee S, Chen L, Loo A, Yang G, Huang A, et al. Single-vector inducible lentiviral RNAi system for oncology target validation. Cell Cycle 2009;8:498-504

33. Singleton DC, Rouhi P, Zois CE, Haider S, Li JL, Kessler BM, et al. Hypoxic regulation of RIOK3 is a major mechanism for cancer cell invasion and metastasis. Oncogene 2015;34:4713-22.

34. Ullah MS, Davies AJ, Halestrap AP. The plasma membrane lactate transporter MCT4, but not MCT1, is up-regulated by hypoxia through a HIF- $1 \alpha$ dependent mechanism. J Biol Chem 2006;281:9030-7.

35. Rios EJ, Fallon M, Wang J, Shimoda LA. Chronic hypoxia elevates intracellular $\mathrm{pH}$ and activates $\mathrm{Na}+/ \mathrm{H}+$ exchange in pulmonary arterial smooth muscle cells. Am J Physiol Lung Cell Mol Physiol 2005;289:L867-74.

36. Wykoff CC, Pugh CW, Maxwell PH, Harris AL, Ratcliffe PJ. Identification of novel hypoxia dependent and independent target genes of the von HippelLindau (VHL) tumour suppressor by mRNA differential expression profiling. Oncogene 2000;19:6297-305.

37. Parks SK, Chiche J, Pouyssegur J. Disrupting proton dynamics and energy metabolism for cancer therapy. Nat Rev Cancer 2013;13:611-23.

38. Vaupel P, Hockel M, Mayer A. Detection and characterization of tumor hypoxia using pO(2) histography. Antioxid Redox Signal 2007;9:1221-35.

39. Carroll VA, Ashcroft M. Role of hypoxia-inducible factor (HIF)-1 $\alpha$-versus HIF- $2 \alpha$ in the regulation of HIF target genes in response to hypoxia insulin-like growth factor-1, or loss of von Hippel-Lindau function: Implications for targeting the HIF pathway. Cancer Res 2006;66:6264-70.

40. Moullan N, Mouchiroud L, Wang X, Ryu D, Williams EG, Mottis A, et al Tetracyclines disturb mitochondrial function across eukaryotic models: call for caution in biomedical research. Cell Rep 2015 Mar 10. [Epub ahead of print].

41. Gawenis LR, Bradford EM, Prasad V, Lorenz JN, Simpson JE, Clarke LL, et al. Colonic anion secretory defects and metabolic acidosis in mice lacking the NBC1 Na+/HCO3 - cotransporter. J Biol Chem 2007;282:9042-52.

42. Igarashi T, Inatomi J, Sekine T, Cha SH, Kanai $Y$, Kunimi M, et al. Mutations in SLC4A4 cause permanent isolated proximal renal tubular acidosis with ocular abnormalities. Nat Genet 1999;23:264-6.

43. Jin L, Sugiyama H, Takigawa M, Katagiri D, Tomitori H, Nishimura K, et al. Comparative studies of anthraquinone- and anthracene-tetraamines as blockers of N-methyl-D-aspartate receptors. J Pharmacol Exp Ther 2007; 320:47-55

44. Groger N, Vitzthum H, Frohlich H, Kruger M, Ehmke H, Braun T, et al Targeted mutation of SLC4A5 induces arterial hypertension and renal metabolic acidosis. Hum Mol Genet 2012;21:1025-36.

45. Bok D, Galbraith G, Lopez I, Woodruff M, Nusinowitz S, BeltrandelRio $\mathrm{H}_{\text {, }}$ et al. Blindness and auditory impairment caused by loss of the sodium bicarbonate cotransporter NBC3. Nat Genet 2003;34:313-9.

46. Chambrey R, Kurth I, Peti-Peterdi J, Houillier P, Purkerson JM, Leviel F, et al. Renal intercalated cells are rather energized by a proton than a sodium pump. Proc Natl Acad Sci U S A 2013;110:7928-33.

47. Jacobs S, Ruusuvuori E, Sipila ST, Haapanen A, Damkier HH, Kurth I, et al Mice with targeted Slc4a10 gene disruption have small brain ventricles and show reduced neuronal excitability. Proc Natl Acad Sci U S A 2008;105: 311-6.

48. Damkier HH, Praetorius J. Genetic ablation of Slc4a10 alters the expression pattern of transporters involved in solute movement in the mouse choroid plexus. Am J Physiol Cell Physiol 2012;302:C1452-9.

49. Cazares-Korner C, Pires IM, Swallow ID, Grayer SC, O'Connor LJ, Olcina $\mathrm{MM}$, et al. CH-01 is a hypoxia-activated prodrug that sensitizes cells to hypoxia/reoxygenation through inhibition of Chk1 and Aurora A. ACS Chem Biol 2013;8:1451-9.

50. Robey IF, Baggett BK, Kirkpatrick ND, Roe DJ, Dosescu J, Sloane BF, et al. Bicarbonate increases tumor $\mathrm{pH}$ and inhibits spontaneous metastases. Cancer Res 2009;69:2260-8. 